\title{
Norois
}

Environnement, aménagement, société

$226 \mid 2013$

Commerce et développement local, déplacements urbains, valorisations agricoles, découpages électoraux

\section{Les Appellations d'Origine Contrôlée (AOC) viticoles dans la région Centre-Val de Loire et les châteaux : une impossible équation?}

Guaranteed vintages in Centre and Val de Loire regions and castles: an

impossible equation?

François Legouy et Christophe Vitré

\section{(2) OpenEdition}

\section{Journals}

Édition électronique

URL : http://journals.openedition.org/norois/4595

DOI : $10.4000 /$ norois. 4595

ISBN : 978-2-7535-2287-9

ISSN : $1760-8546$

\section{Éditeur}

Presses universitaires de Rennes

Édition imprimée

Date de publication : 30 mars 2013

Pagination : 63-77

ISBN : 978-2-7535-2285-5

ISSN : 0029-182X

\section{Référence électronique}

François Legouy et Christophe Vitré, « Les Appellations d'Origine Contrôlée (AOC) viticoles dans la région Centre-Val de Loire et les châteaux : une impossible équation? », Norois [En ligne], 226 | 2013, mis en ligne le 30 mars 2015, consulté le 01 mai 2019. URL : http://journals.openedition.org/ norois/4595; DOI : 10.4000/norois.4595 


\title{
Les Appellations d'Origine Contrôlée (AOC) viticoles dans la région Centre-Val de Loire et les châteaux : une impossible équation?
}

\author{
Guaranteed Vintages in Centre and Val de Loire Regions and Castles: \\ An Impossible Equation?
}

\author{
François Legour ${ }^{* a}$, Christophe VITRÉb
}

\footnotetext{
*Auteur correspondant

a Maître de Conférences, directeur du département de Géographie, EA 1210 - CEDETE, Université d’Orléans,

10 rue de Tours, BP 46527, 45065 ORLÉANs Cedex 2 (francois.legouy@univ-orleans.fr)

b Professeur certifié au Lycée de Nogent le Rotrou(christophe.vitre@gmail.com)
}

Résumé : Comparé à la région bordelaise, le château en région Centre - Val de Loire n'est pas ou qu'exceptionnellement une exploitation viticole. Et pourtant, les authentiques châteaux sont sans doute plus nombreux ici que partout en France, comme l'histoire des vignobles de la région est liée à ces mêmes châteaux. L'explication historique telle que Roger Dion l'a éclairée, fait apparaître un processus de dé-identification territoriale à partir du XVII ${ }^{\mathrm{e}}$ siècle. Nous sommes actuellement à un moment clé en France où l'œnotourisme apparaît comme une possibilité de développement territorial, lequel passe par un processus de valorisation patrimoniale. Il est ainsi possible de mettre en lumière une timide contiguïté spatiale entre château et vigne, d'abord toujours conservée comme à Valençay et Chinon, entre autres exemples, mais aussi un processus de ré-appropriation territoriale en reliant le château à la vigne, comme cela est manifeste à Cheverny. En effet, l'AOC Cheverny y a pris en 1993 le nom du château éponyme et l'image du château est souvent reproduite sur les étiquettes des bouteilles. Surtout, les viticulteurs ont installé en 2008 une maison des vins à la sortie du château, maison qui rencontre un véritable succès s'il faut en croire la fréquentation touristique et le volume des bouteilles vendues sur place.

Abstract: In comparison with the Bordeaux wine region, a chatean in the region Centre-Loire Valley is never, or only exceptionally, a wine estate. Nevertheless, there are in this region more authentic chateaux than anywhere else in France and the history of the vineyards is linked to these chateaux. Roger Dion explained this historical process by a territorial de-identification since the 17th century. Now is a key period in France as wine tourism offers possibilities for territorial development which requires heritage re-appropriation. The development of spatial proximity between chateaux and vineyards can be emphasized where it never disappeared like in Valençay or in Chinon and also through the territorial re-appropriation connecting chateaux and vineyards, like in Cheverny. Indeed, in 1993, the Cheverny AOC took the name of the chateau and the image of the castle is often reproduced on bottles' labels. Also, in 2008, wine growers have opened a wine shop at the exit of the castle that has been a real success according to the number of tourist visits and bottles sold there.

Mots clés : « château viticole » - valorisation patrimoniale - identité territoriale - œnotourisme - modèle paysager

Keywords: chateau - heritage validation - territorial identity - wine tourism - landscape model 


\section{INTRODUCTION}

La région Centre - Val de Loire compte en janvier 201225 AOC viticoles. S’il est une région qui concentre le plus de châteaux en son sein, c'est bien cette région qui longe le Val de Loire, particulièrement à l'aval d'Orléans, avec entre autres exemples, les châteaux de Chambord, de Blois et de Cheverny qui sont sans doute les plus connus et les plus visités. Il est donc facile de relier Val de Loire et châteaux. De fait, Roger Dion (1934) avait rappelé que le vocable du Val de Loire avait ses origines dans l'Orléanais et s'était ensuite propagé jusqu'à Nevers et aux alentours de Blois, puis à l'ensemble de la vallée de la Loire. Pourtant, de nos jours et dans les paysages, le lien entre les châteaux et les vignobles n'est pas évident à première vue en ces régions contrairement au Bordelais, où la liaison est ancienne et efficace. Selon Philippe Roudié (2000), elle remonte à la mi-XIX siècle pour s'imposer peu à peu entre 1860 et 1890 et éliminer le terme de cru ou de domaine alors plus usité. Tout le monde sait que les exploitations viticoles sont, en cette région, des " châteaux », quand bien même les bâtiments n'en auraient aucunement l'apparence architecturale. Pour Michel Réjalot (2003, 2011), à l'origine, le « château » bordelais est une résidence champêtre, forme d'investissement de la noblesse et de la bourgeoisie dans la terre, pour vivre ensuite de ses rentes, sorte de "villa romaine " après l'heure. Puis, « le terme de château est devenu une appellation qui ne représente plus nécessairement une construction castrale mais un domaine viticole ».

Le château viticole représente donc le symbole fort du vignoble bordelais. Il possède une connotation méliorative, anoblissante et relève également de l'imaginaire territorial inscrit dans la représentation architecturale. Le vin est un produit noble, il est logique que les bâtiments du vin relèvent de cette noblesse.

Pas ou très peu de " châteaux viticoles » au sens d'exploitation viticole sont présent en région Centre - Val de Loire. P. Roudié (2000) pousse l'analyse jusqu'à affirmer :

"Aussi est-ce vers la Loire qu'il faut se tourner pour trouver quelques nouveaux "châteaux" au pays du «muscadet », des "coteaux du Layon" ou des "Saumur Champigny", poussant même une pointe timide jusqu'en Sancerrois. Mais, par un mimétisme (inconscient?) avec le Bordelais, ces "châteaux" viticoles ne sont évidemment pas les fameux châteaux de la Loire soit urbains (Blois, Amboise...) ou champêtres et forestiers (Chambord, Chenonceau...) ou décor de théâtre et jardin (Villandry, Cheverny...) où la vigne n'a jamais eu sa place. »

La dernière phrase de la citation est à nuancer. La vigne, en effet, a été très présente dans le Val de Loire, et ce, jusqu'à une date relativement récente à l'échelle de l'histoire contemporaine, mais pas toujours en lien avec des châteaux et les surfaces viticoles ont fortement décru, comme en d'autres régions. De nos jours, la région, par le Val de Loire, a renoué avec son vignoble et ses châteaux; en témoigne son rattachement au patrimoine mondial de l'Humanité par l'Unesco en 2000 (Bonté, 2009). Par contre, et malgré quelques mises en scènes paysagères à visée œnotouristique la liaison «vigne - vin - œnotourisme - château » ne fonctionne encore que trop timidement, ici comme en de maints endroits en France, ainsi que l'a souligné avec justesse Sophie Lignon-Darmaillac (2009) :

« Alors qu'une route du vin sillonne la Bourgogne et la Champagne depuis 1934, l'Alsace depuis 1953, alors que de nombreuses foires aux vins ou fêtes vigneronnes animent chacun des vignobles français, le tourisme œnologique ou œnotourisme ne s'est généralisé en France que récemment, dans les années 1990. Les routes touristiques étaient alors réservées aux châteaux, églises ou abbayes, comme autant d'itinéraires culturels pour des touristes curieux de découvrir un patrimoine architectural d'exception. »

Pourtant, certains auteurs (Péron, 2001) insistent sur le lien développement territorial et patrimoine :

«Dans ces stratégies territoriales, les éléments patrimoniaux sont considérés comme des richesses à la fois économiques (le tourisme qui en découle) et symboliques (l'image rajeunie de l'espace local) non négligeables que les élus locaux cherchent désormais à inventorier, à investir, à développer ou à créer. »

Il est donc possible de postuler comme hypothèse de départ que le processus de territorialisation par la patrimonialisation (Senil, 2011), du moins en ce 
qui concerne le monde viticole et ses relations avec les châteaux en est encore à ses balbutiements, mais jusqu'à quel point? Peut-on réaliser un état des lieux et de la dynamique des relations entre vignes et châteaux dans la région Centre-Val de Loire? Quelle est l'importance théorique des liens patrimonialisation - territorialisation?

Pour répondre à la problématique et au questionnement, la méthode consistera d'abord en l'analyse des paysages castraux et viticoles et à leur association, à identifier leur modèle paysager historique, au dénombrement des « châteaux viticoles » pour deux départements témoins, à rappeler la tendance actuelle du développement territorial par le biais de la valorisation patrimoniale, et à présenter des exemples plus ou moins aboutis de prise en compte du château dans la mise en valeur de la vigne et du vin.

C'est pourquoi, il sera d'abord présenté un état des lieux, à la fois géographique et historique, des liens socio-économiques, culturels entre vignoble et patrimoine castral, dans la région Centre - Val de Loire, dans un mouvement d'identification puis de dé-identification territoriale. Dans un deuxième temps, au contraire, il sera insisté sur le passage du développement territorial par le patrimoine et sur les progrès réalisés pour renouer la relation vignoble patrimoine castral. Enfin, avec l'exemple particulier de l'appellation d'origine contrôlée Cheverny, nous verrons à quel point on assiste à un retour en force des châteaux dans leurs liens étroits avec la vigne avec une ré-identification territoriale, mais encore incomplète et imparfaite.

\section{LES LIENS GÉOGRAPHIQUES ET HISTORIQUES ENTRE VIGNOBLES ET CHÂTEAUX DANS LA RÉGION Centre - Val de Loire}

\section{Un vignoble lié aux cours d'eau et aux châteaux qui les contrôlent}

La vigne reste prégnante pour qui la regarde dans la région Centre - Val de Loire à laquelle a été ajoutée le département de la Nièvre qui, bien que situé en Bourgogne, est rattaché traditionnellement par l'INAO ${ }^{1}$ au Val de Loire. Au total, six départements avec près de 6000 exploitations viticoles

1. INAO : Institut National de l'Origine et de la Qualité pour environ 24000 ha de vignes en $2000^{2}$ (figure 1 - planche VII).

Le vocable Val de Loire insiste sur la forte présence de la vigne le long de la vallée de la Loire et de ses affluents qui, de fait, accaparent l'essentiel du vignoble régional avec les vignobles, souvent les plus réputés, de Pouilly-Fumé, Sancerre, Coteaux du Giennois, Orléans, Cheverny, Cour-Cheverny, des diverses déclinaisons des vins de Touraine, Vouvray, Bourgueil, Montlouis, Chinon, Coteaux du Vendômois. Autant de vignobles produisant du vin de qualité apparaissant comme canalisés par les vallées, principaux couloirs de communications qui, jusqu'à l'avènement du chemin de fer, permettaient un commerce au loin. Une autre partie du vignoble est située le long d'une ligne qui prend en écharpe le Berry selon une direction est-nord-est/ouestsud-ouest, avec Sancerre, Menetou-Salon, Quincy, Reuilly, Valençay, alignement perpendiculaire au sens d'écoulement des affluents principaux de la Loire au sud du fleuve qui correspond à la limite entre le crétacé au nord et le jurassique au sud. Un peu à part, est le vignoble de Chateaumeillant, au sud du Berry. Enfin, un vignoble plus diffus, très dispersé est disséminé dans l'ensemble de la région et qui provient de l'héritage d'un vignoble de consommation courante et familiale, fortement présent dans le département de l'Indre et au sud du département de l'Indre et Loire, beaucoup moins au nord de la Loire.

Ces vignobles correspondent à deux héritages historiques dont R. Dion (1952 et 1959) a très bien décrit la distribution géographique et leur genèse : les vignobles populaires et les vignobles de qualité. Les premiers sont cultivés par les populations paysannes, partout où c'est possible, que ce soit en concurrence avec les céréales sur les plateaux ou dans les zones inondables des cours d'eau, en particulier dans le Val de Loire, au détriment des prairies, évidemment sur les versants et talus voués usuellement aux vergers et à la vigne.

Dans l'ensemble, il s'agit de vignes qui sont d'un rapport intéressant pour le paysan, mais fournissant un vin de piètre qualité. Il en est tout autrement des vignobles produisant des vins de qualité. Pour

\footnotetext{
2. Ce chiffre de 6000 exploitations viticoles provient du Recensement Général Agricole de 2000 et doit être compris au sens large, y compris les exploitations détenues par des retraités de l'agriculture qui ont la possibilité de conserver quelques arpents de vignes au moment de leur retraite.
} 
R. Dion, ces derniers ne peuvent se comprendre s'il n'est pas fait référence aux modes de transports par les voies navigables jusqu'à l'arrivée du chemin de fer et/ou si on ne considère pas le propriétaire du vignoble, évêque, moine, prince, noble et seigneur, bourgeois, catégories à resituer sous l'ancien Régime, et qui ont été réduites majoritairement à la viticulture bourgeoise et celle pratiquée par des paysans aisés après la Révolution (Combaud et Legouy, 2010). Ces personnages ont eu à cœur de produire tout au long de l'histoire des vins dignes de leur rang et qui étaient à la fois un symbole de leur position sociale, un hommage à leurs invités de marque et un produit capable d'être vendu fort cher et fort loin. Le vin de qualité est donc un produit anoblissant lié à un personnage qui détient un pouvoir spirituel et/ ou temporel, une autorité, une notoriété ou qui tend à s'en rapprocher.
Or et malgré les apparences actuelles, il est possible, historiquement parlant, de rapprocher vignes, vins de qualité, châteaux et villes. En effet, les principales villes de la région sont originellement des bourgs castraux et sont souvent situées en des lieux névralgiques par rapport aux voies de communications, sur des gués (villes-ponts de Tours, Amboise, Chaumont-sur-Loire, Blois, Beaugency, Meungsur-Loire, Orléans...), en position haute (Sancerre en est sans doute le meilleur exemple) et/ou des confluences de cours d'eau (Chinon). Leur situation géographique n'est donc jamais anodine. Ces villes avec châteaux étaient alors souvent accompagnées de vignes (Dion, 1952) :

« La vigne en effet [...] tenait, dans les préoccupations de nos ancêtres, une telle place que quiconque, parmi eux, avait un rang, la cultivait pour l'honneur, autant que pour le profit, en sorte que

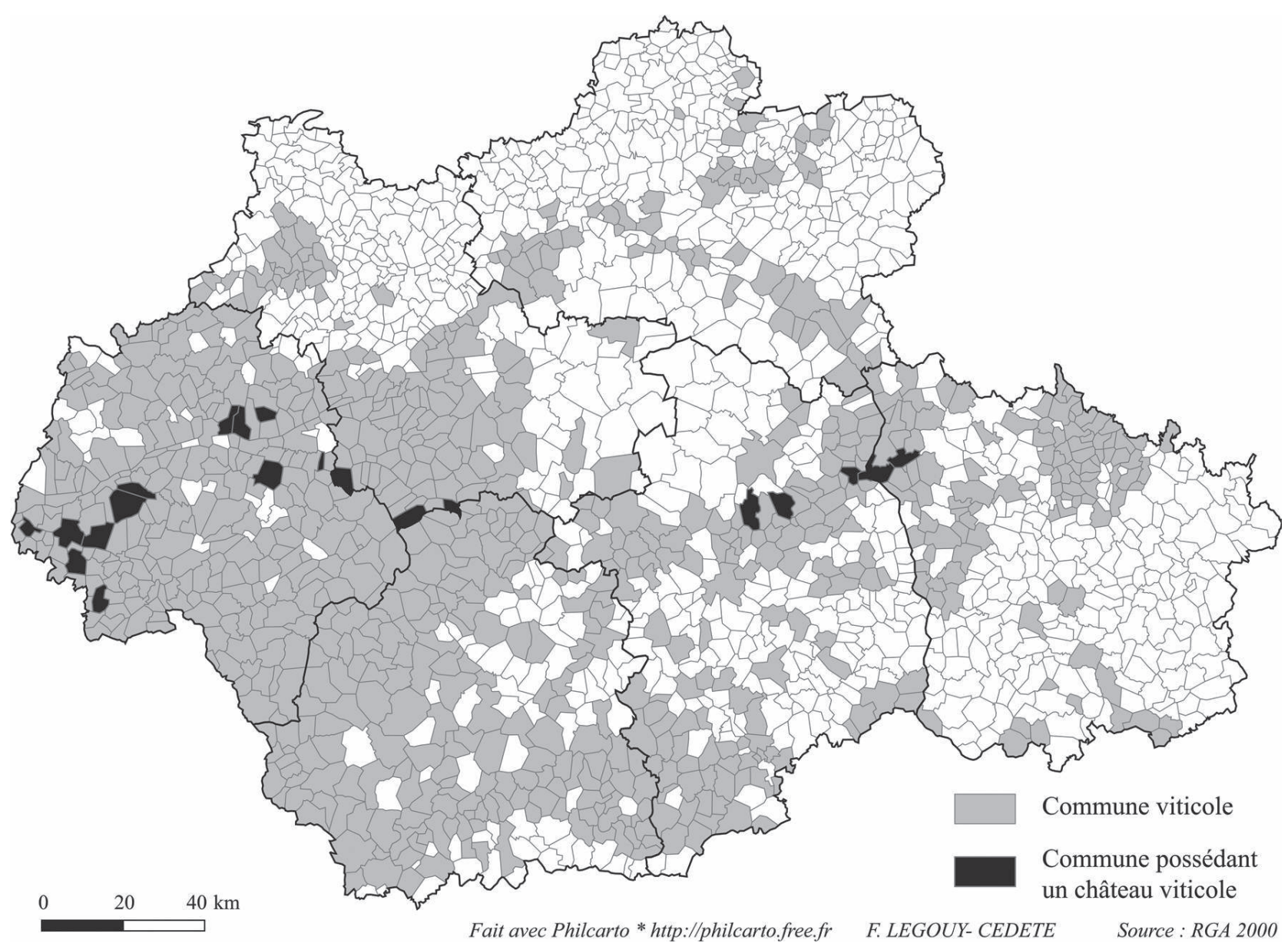

Figure 2 : Communes et châteaux viticoles de la région Centre-Val de Loire (RGA 2000). Towns and chateaux in the region Centre-Val de Loire (RGA 2000). 
la seule présence d'un château, ou d'une bourgade rehaussée de quelques maisons opulentes, suffisait à faire paraître un vignoble [...]. Limitée en bien des lieux, au seul clos seigneurial, cette viticulture de prestige n'en marquait pas moins, par le grand nombre de ses apparitions, le paysage de l'ancienne France. [...] Présente, ou tout au moins désirée, dans tous les lieux où paraissent le luxe ou les signes de l'opulence, la vigne, celle qu'on cultive pour servir la bonne renommée d'une maison, est, dans la France médiévale, et reste, jusqu'à la fin de l'Ancien Régime, l'ornement ordinaire des villes. »

Ainsi, la noblesse d'un lieu et d'un personnage se traduit dans le paysage par la présence de vignes encloses, souvent dans une grande proximité spatiale, comme au pied des remparts. Ce modèle paysager vitivinicole a traversé les siècles et a perduré, mais très inégalement et beaucoup plus à Bordeaux que dans le Val de Loire. De nos jours, le lien vigne - château reste nettement visible en quelques rares endroits comme à Chinon ou à Valençay. En ce dernier lieu, la vigne est effectivement enclose dans la parcelle de la "basse-cour », en contrebas du château (figure 3).

Ce modèle vitivinicole castral repose sur des images célèbres. Parmi ces images, les enluminures des Très riches heures du Duc du Berry sont certainement les plus caractéristiques. Ces enluminures sont rassemblées dans un manuscrit du début du $\mathrm{Xv}^{\mathrm{e}}$ siècle dont les plus connues représentent des décors médiévaux mêlant des scènes paysannes et seigneuriales en fonction du calendrier et des saisons.

Nous en retiendrons deux exemplaires, la séquence du mois de septembre qui montre une scène de vendange à proximité du château de Saumur sur le bord de la Loire et celle du mois de mars avec la taille de la vigne. Cette dernière scène se déroule devant le château de Lusignan dans le Poitou. Le décor des vignes visibles sur les deux enluminures est idéal. Les vignes sont encloses d'un muret de pierres, et sur les extrémités de deux parcelles est blottie une cabane de vignerons ou une tour. Un portail monumental sert d'entrée pour un des clos, et un chemin de terre permet l'accès aux parcelles. Enfin, les clos accueillent quelques arbres, probablement fruitiers, ce qui était très courant avant le labourage par la charrue tirée par le

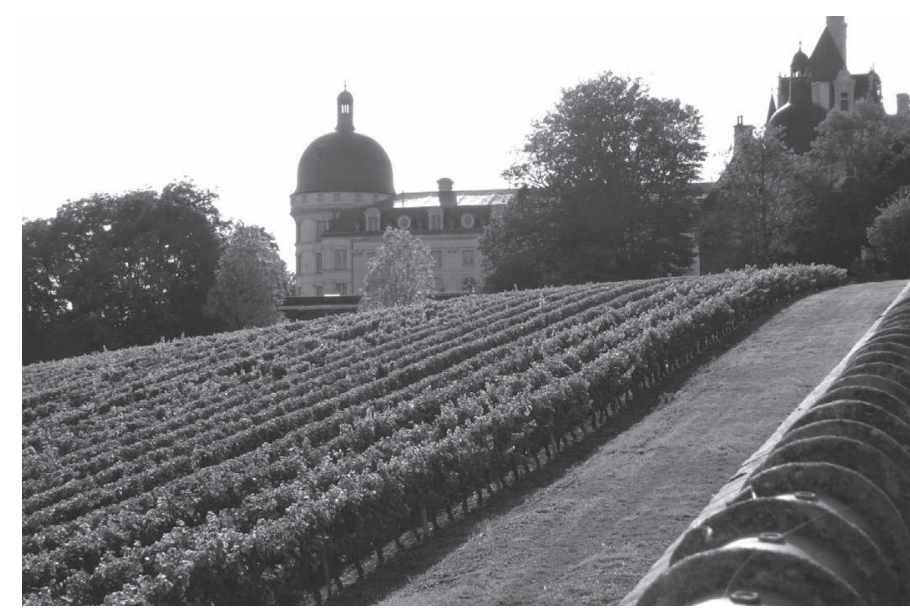

Figure 3 : Le château de Valençay et son vignoble Château of Valençay and its vineyard

cheval. Le travail de la vigne et les outils utilisés y sont très bien illustrés. Ces enluminures décrivent ainsi tous les motifs paysagers traditionnels de la vigne : les pieds de vignes alignés, un clos de murs, une cabane de vigneron, une tour, une entrée monumentale, des arbres fruitiers, un chemin de vigne, sans oublier le château non loin à l'arrière-plan. Tout y est (figures 4 et 5).

Ces enluminures des Très riches heures du Duc de Berry dépeignant la vigne idéale déterminent une synthèse paysagère de la vigne castrale, synthèse qui n'est pas réalisée partout mais qui reste dans la pensée collective comme le modèle paysager viticole par excellence (Bigando, 2006).

La relation entre vigne et château a donc participé à une identification patrimoniale et territoriale. La vigne se mêle au château, comme élément de décor, mais également comme élément de production et de prestige. Elle prend part ainsi à la noblesse et du monument et de son propriétaire. Il s'agit, à l'origine, d'une viticulture princière de qualité. Mais, la relation vigne - château a évolué avec le temps et l'espace.

\section{Un vignoble peu relié aux châteaux en tant qu'exploitations viticoles contraire- ment au modèle bordelais}

Le nombre des châteaux dans la région Centre - Val de Loire est impressionnant. Dans les départements de la Nièvre et du Cher, respectivement plus de 80 et 120 châteaux sont répertoriés sur 


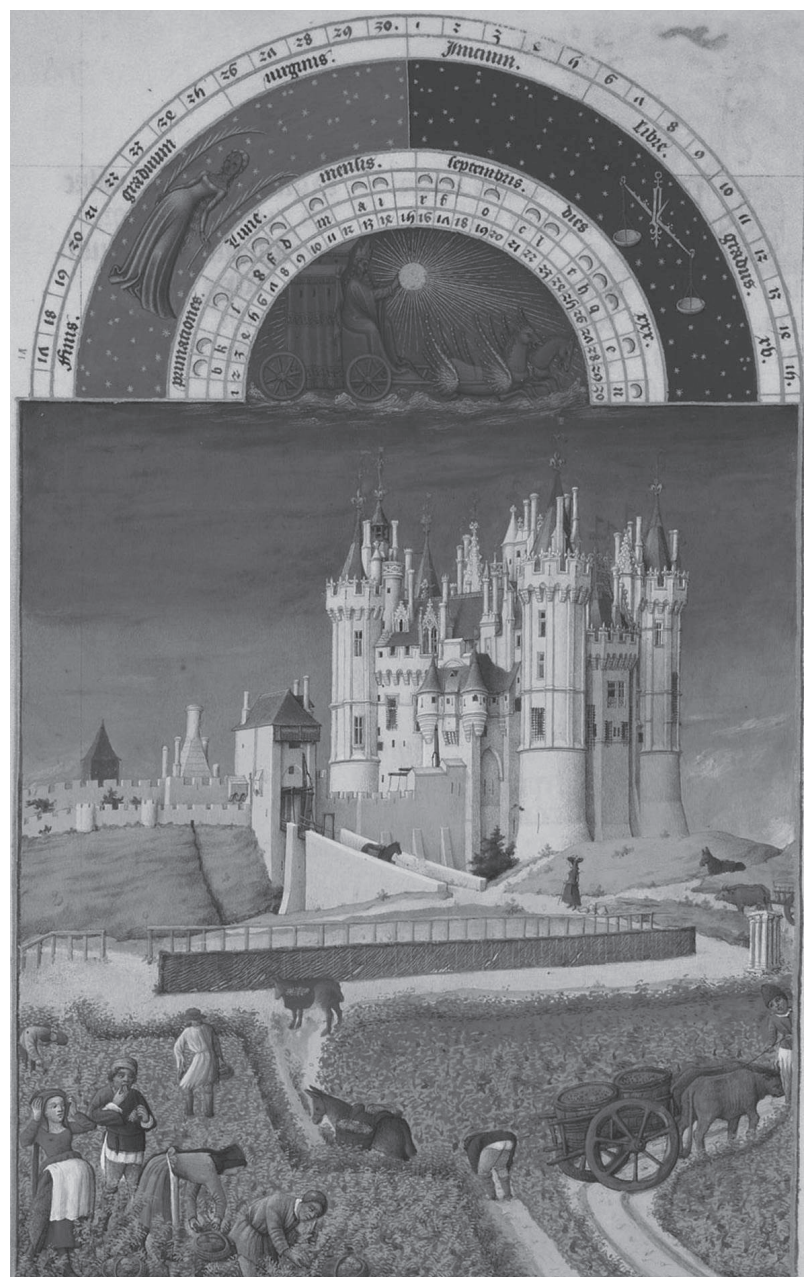

Figure 4 : Le château de Saumur et son vignoble Chateau of Saumur and its vineyard

les cartes $\mathrm{IGN}^{3}$ pour les communes viticoles ${ }^{4}$, ce qui représente plus $50 \%$ et près des $2 / 3$ de communes ciblées comme viticoles possédant un château. Nous sommes bien en phase avec une France des châtellenies qui a marqué son temps et qui est encore visible dans le paysage, (Pinchemel, 1980, p. 226).

Pourtant, malgré ces modèles paysagers et architecturaux qui restent prégnants, malgré une histoire viti-

3. Nous avons utilisé le site Géoportail de l'IGN et avons retenu comme château l'habitat répertorié portant le nom de château et de manoir. Par contre, nous avons délaissé les tours isolées, les pigeonniers, les colombiers, les courtines, les fertés et les mottes qui, bien qu'étant des indicateurs d'une maison seigneuriale, ne correspondent pas pleinement à un habitat castral. Si cela avait été le cas, il aurait fallu augmenter d'un quart environ, le nombre de " châteaux ».

4. Il faut entendre par commune viticole, au sens large, une commune qui détient sur son finage au moins une exploitation viticole, que les vignes soient situées ou non dans les limites administratives communales.

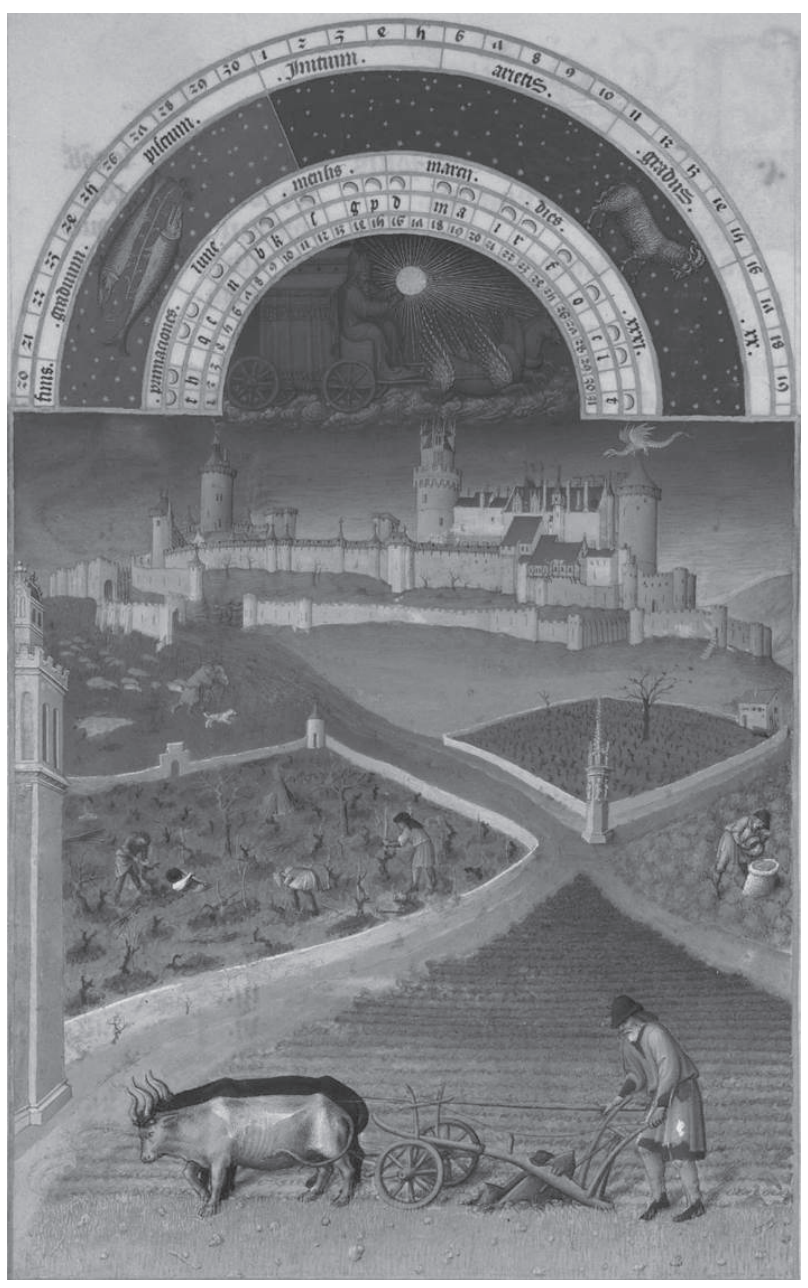

Figure 5 : Le château de Lusignan et son vignoble Chateau of Lusignan and its vineyard

cole fortement marquée par la cour des rois de France qui a longtemps et fréquemment séjourné dans le Val de Loire et dans les régions proches (Pitte, 1990), et qui de fait a promu des vignobles de qualité en son temps, on compte très peu de châteaux viticoles, en tant qu'exploitations viticoles, en région Centre - Val de Loire. Selon les informations fournies par les interprofessions du Val de Loire et Les vins du CentreLoire, il est possible d'estimer le nombre de châteaux viticoles à environ 25 sur plusieurs milliers d'exploitations viticoles en 2012. Dans le département de la Nièvre cité plus haut, sur les 80 châteaux repérés, seuls 3 sont le siège d'une exploitation viticole. Dans le Cher, pas plus de 5 « châteaux » viticoles. Il est vrai que ce nombre est plus important dans le Loiret-Cher et davantage encore dans l'Indre-et-Loire, départements où les châteaux pullulent. Mais nous 
sommes loin du nombre (11000) et du modèle des châteaux viticoles de Bordeaux que Philippe Roudié (2000) a inventorié. Même s'il a bien précisé que certains châteaux viticoles bordelais n'avaient de châteaux que le nom, rien ou si peu de castral dans une architecture qui ressemble au mieux à une demeure bourgeoise de style néo-classique parfois affublée de tours sur les côtés de la maison. Michel Réjalot (2011) rappelle que : "Pétrus, le célèbre cru de Pomerol, ne comporte aucun château au sens castral du terme, mais porte sur ses étiquettes la mention "Mis en bouteille au château"! »

Ici, dans la région, nous sommes à l'opposé du modèle bordelais. Les quelques châteaux viticoles correspondent le plus souvent à l'existence de véritables châteaux ou demeures seigneuriales, antérieures au XIX ${ }^{\mathrm{e}}$ siècle et disposant d'un domaine viticole. Il existe, certes, des châteaux qui ressemblent davantage à des demeures bourgeoises s'approchant du modèle castral par la présence d'une tour accolée à la maison et/ou d'un pigeonnier, marque du privilège seigneurial. «Château » Quinçay à Meusnes, à la limite du vignoble de Valençay et de la Touraine, en est un très bon exemple. Cependant, il est relativement rare d'observer des vignes au pied des murs ou des remparts. Le plus souvent, le « château » viticole est entouré par des jardins, fréquemment façonnés à la française, et par des prés. Il faudrait sans doute nuancer le propos en précisant qu'à proximité de Tours, il existe bien des châteaux non loin des vignes (Château de Jallanges à Vernou sur Brenne, château d'Anzan à Noizay, Château de Moncontour à Vouvray, par exemple, ce dernier étant bien un château vitivinicole). Pourtant, les vignes se retrouvent toujours décalées dans l'espace et séparées par un tampon de jardin et de verdure. Le modèle paysager château - vignes est donc très minoritaire. Les exemples de Valençay et de Chinon restent l'exception.

Comment comprendre alors cette perte ou cette absence de modèle vigne - château qui a été promu par ailleurs à Bordeaux?

À Bordeaux, le vocable de « château » a été abondamment utilisé pour apporter un supplément de noblesse à un produit qui ne l'est pas moins. De fait, la stratégie utilisée en force à partir de la seconde moitié du XIX ${ }^{e}$ siècle correspond à du marketing territorial : le « château » est une véritable marque, quasi supérieure à l'AOC de nos jours. Et nombre d'exploitations viticoles ou de demeures bourgeoises

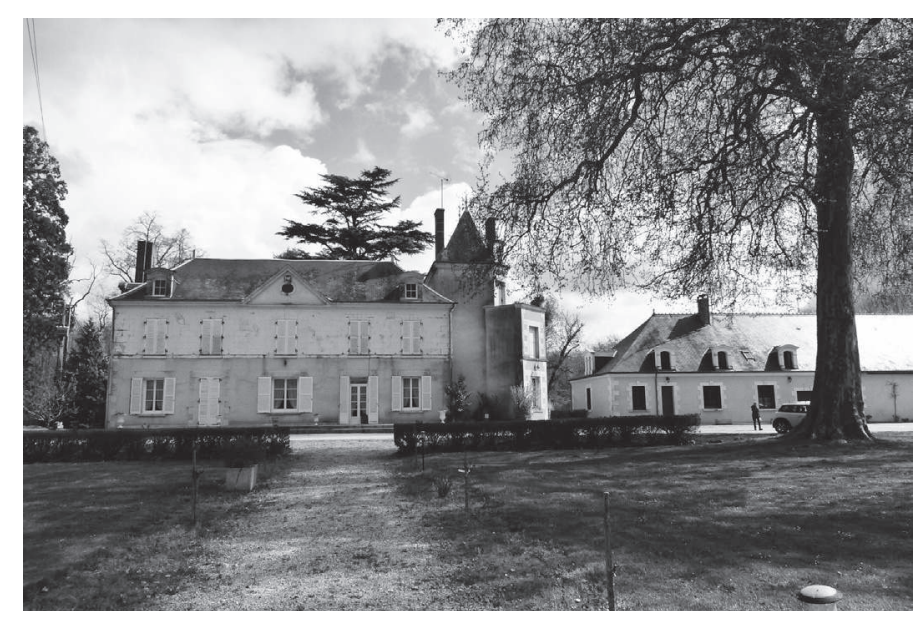

Figure 6 : Château Quinçay à Meunes (41)

Chateau of Quinçay in Meunes (41)

dans leur architecture, centres d'exploitation viticole ont été ainsi « anoblies ».

Concernant la région Centre - Val de Loire, il est possible de reprendre l'argumentation de Roger Dion qui a montré comment la transition a été menée. Les châtelains, progressivement au cours du XVII ${ }^{\mathrm{e}}$ siècle et au-delà, ont été détournés de leurs préoccupations viticoles et des soins qu'ils devaient apporter à leurs vignes, par d'autres intérêts :

«Ruine des murailles, et aussi des traditions : cette viticulture attachée au château, que le Moyen Âge avait connue exemplaire et audacieuse, perdit de sa force et de son excellence quand le seigneur, retenu à la cour, ne fut plus là pour veiller personnellement sur elle. [...] Â cet égard, ce sont des changements expressifs que ceux qui se manifestent dans l'aspect de la demeure seigneuriale, et plus précisément dans le caractère de sa décoration végétale, au cours de la période qui va des derniers siècles du Moyen-Âge à la fin de l'Ancien Régime. [...] au Moyen-Âge, [...] Il était rare alors que, du haut des remparts de la ville ou des murs du château, on ne pût découvrir du regard quelque importante partie. [...] Au XVI ${ }^{\mathrm{e}}$ siècle encore, le clos seigneurial continue de se montrer aux visiteurs du château à côté du jardin dessiné à l'italienne et c'est seulement sous Louis XIV qu'on le verra s'effacer pour faire place à des aménagements d'un autre style. [...]» (Dion, 1959).

Ainsi, en région Centre - Val de Loire, le château viticole a-t-il été abandonné, comme la cour 
royale a délaissé le Val de Loire. Les nobles ont eu d'autres préoccupations. Le modèle paysager et marketing du " château - vignoble ", ainsi que l'équation château = exploitation viticole n'ont pas été retenus, encore moins l'équation château = appellation! Nous sommes dans un processus de déconstruction ou de dé-identification de la vigne au château. Par ce processus, le décor viticole n'est plus celui des châteaux, mais celui plus quelconque de simples exploitations agricoles car la viticulture en cette région a été en partie accaparée par les paysans qui y ont vu tout leur intérêt. Ce phénomène a commencé dès le $\mathrm{XVII}^{\mathrm{e}}$ siècle et s'est généralisé après la Révolution française. Il a été marqué, entre autres, par l'abandon relatif des cépages donnant des vins fins (auvernat) et au contraire, l'utilisation de cépages à fort rendements (gamays, teinturiers, hybrides depuis la crise du phylloxéra et la restauration du vignoble à la fin du XIX ${ }^{\mathrm{e}}$ siècle) et produisant des vins de consommation plus courante pour l'approvisionnement du marché parisien en vins.

Le mouvement de balancier entre les deux régions, bordelaise et Centre - Val de Loire peut apparaître paradoxal. Il se comprend si on considère que la région bordelaise a toujours été une grande région exportatrice de vins de qualité sous l'influence du marché anglais et que les Bordelais ont fait le choix de la qualité très tôt (à partir du XvII ${ }^{\mathrm{e}}$ siècle), en vue justement de l'exportation, ce qui n'a pas été autant le cas du Val de Loire, particulièrement à l'amont de Blois (Legouy, 2012). Le virage vers une production de vins de qualité pour une exportation massive, dans le Centre-Val de Loire, est intervenu plus tard, sauf peut-être pour certains vignobles aux surfaces restreintes (Vouvray et Montlouis par exemple).

Et pourtant, paradoxe, l'identité territoriale de la région est fortement marquée par les châteaux.

\section{LES AVANCÉES DE LA TERRITORIALI- SATION PAR LA PATRIMONIALISATION dans LA RÉGion CENTRE - VAL DE LOIRE}

\section{Les enjeux de la territorialisation par la patrimonialisation et l'œnotourisme}

« Patrimoines, paysages, art de vivre constituent le triptyque d'atouts majeurs de l'offre touristique de la France. Les paysages de vignes, la culture historique et les atouts patrimoniaux de l'activité viticole, la culture du vin et du savoir « bien » vivre emblématique de la France. » (Dubrule, 2007)

Cet extrait du rapport de Paul Dubrule sur l'œnotourisme dessine bien les enjeux de la valorisation du patrimoine. Qui dit valorisation, dit d'abord identification de ce patrimoine lié à la vigne et au vin. Dans quelle mesure, cette patrimonialisation peutelle entraîner de la territorialisation puis du développement territorial?

La mise en évidence du patrimoine doit d'abord passer par un processus d'appropriation du territoire, donc une prise de conscience, par ses habitants, avec lequel ses habitants ont des liens, affectifs et culturels privilégiés. Ces liens sont sans doute encore plus intenses avec les éléments rappelant cette identification et cette vision à la fois individuelle et collective, en particulier le patrimoine qui donne une identité à un territoire et lui permet de le distinguer des autres territoires. Le patrimoine rural, a rappelé Isac Chiva, est constitué des paysages, des immeubles, des produits du terroir, des techniques, des outils et des savoir-faire formant un ensemble cohérent et indissociable (Chiva., 1994).

Surtout, territoire et patrimoine sont liés par une identité, un passé et un avenir communs pour une population qui, du fait de la tradition et d'un « vivre ensemble ", accepte de s'y reconnaître. Vouloir développer un territoire, c'est mettre en évidence ses forces et ses atouts, malgré les contraintes et les menaces, dans une perception partagée par ses acteurs. De ces atouts, incontestablement le patrimoine est fondamental car il détermine une communauté de destin et l'espace d'une mémoire partagée. Développer un territoire, c'est donc, notamment pour les territoires vitivinicoles, faire émerger une identité territoriale, et faire resurgir une tradition et des usages qui ont su évoluer avec le temps. Les produits de terroirs, dont le vin, sont représentatifs de cette reconnaissance (Unesco, 2005).

"Les terroirs sont des espaces géographiques délimités, définis à partir d'une communauté humaine qui construit au cours de son histoire un ensemble de traits culturels distinctifs, de savoirs et de pratiques fondés sur un système d'interactions entre le milieu naturel et les facteurs humains. [...] Les savoir-faire mis en jeu révèlent une originalité, confèrent une typicité et 
permettent une reconnaissance pour les produits ou services originaires de cet espace et donc pour les hommes qui y vivent. » (Collectif, 2005)

Ici, le mot terroir rejoint le mot territoire : un produit de terroir est un produit de territoire ainsi que le rappelle à l'envi les produits de marque investis dans le terroir et qui rassurent le consommateur à la recherche d'authenticité, de saveurs renouvelées et à des aspirations épicuriennes (Rastoin, 2004).

Au-delà des produits de terroir, les enjeux sont fondamentaux. C'est toute la justification des labels de qualité, en particulier des AOC françaises mises à l'heure européenne avec les AOP, en 2007-2009 pour une défense commune d'une agriculture coûteuse, mais de qualité, face aux produits agro-industriels des pays du Nouveau Monde qui voient dans ces labels une protection déguisée et une entorse à la concurrence et au libre marché, sans que la qualité ne soit totalement garantie (Legouy, 2007; Sylvander, 2005).

Ces enjeux sont encore renforcés par l'approfondissement d'une crise vitivinicole depuis 2000 entraînant des remises en causes pour certains vignobles produisant des vins de bas et de moyen de gamme qui perdent des parts de marché, à la fois à l'échelle nationale par la baisse de la consommation et à l'échelle internationale du fait de la concurrence exacerbée des pays du Nouveau Monde. La crise était présente pour les vins de table depuis les années 1970-1980, elle a gagné certaines appellations régionales au cours des années 1990-2000 (Legouy, 2008). Afin d'essayer d'endiguer la crise, la réflexion a été de développer l'œnotourisme et donc de mettre en valeur le patrimoine vitivinicole. L'idée était que pour fidéliser la clientèle française comme étrangère, il fallait l'attirer sur son territoire, en montrer toutes les aménités, la faire déguster et espérer que le «miracle » de la «madeleine de Proust » ferait encore son effet : boire un vin de qualité dans un paysage de qualité laisse des souvenirs ineffaçables. En d'autres termes, il fallait remettre à l'honneur les routes des vins créées pour quelques unes dès les années 1930-1950, au moment où la crise économique sévissait ou au moment des trente glorieuses où on assistait à un redécollage de l'économie vitivinicole. Routes des vins? Bien plus en définitive. Le rapport Dubrule souligne la diversité des activités relevant de l'œnotourisme : 1. routes des vins; 2. sentiers et chemins de randonnées viticoles; 3. circuits ou séjours organisés dans le vignoble; 4. stages d'œnologie; 5 . visites et autres prestations des exploitations viticoles; 6. musées, écomusées du vin; 7. maisons du vin; 8 . fêtes et festivals; 9. salons professionnels, foires au vin locales, ventes aux enchères.

Ces diverses prestations œnotouristiques englobent la mise en valeur des paysages viticoles, le petit patrimoine rural et les châteaux qui en sont l'élément le plus emblématique. Il s'agit en l'occurrence d'un processus de ré-identification qui doit déboucher sur une re-territorialisation par la patrimonialisation. Le patrimoine est donc le symbole d'un territoire pour les yeux des acteurs intérieurs du territoire et des spectateurs extérieurs : les touristes, ainsi que le montrent les deux exemples suivants.

\section{Patrimoine castral et onotourisme en région centre val de loire : deux exemples}

Dans quelle mesure, l'élément patrimonial castral est-il mis à contribution pour mettre en valeur le vignoble et le vin dans la région Centre - Val de Loire?

Parmi les facteurs de re-territorialisation par la patrimonialisation, il est possible de mettre en avant les représentations de mises en scènes paysagères œnotouristiques présentées à l'œil du touriste qui traverse la région tourangelle par l'intermédiaire de panneaux le long de l'autoroute qui amène sur Tours depuis Paris. On y voit, sur l'un d'entre eux, avant l'entrée dans l'agglomération tourangelle, au premier plan une grappe de raisin, en plan central des rangs de vignes et à l'arrière plan un château. Nous sommes pour la région dans l'«image d'Épinal » ciblant la mémoire collective. Pour être certain, que le diptyque vigne - château aura été retenu, quelques pieds de vignes ont été effectivement disposés à proximité immédiate des châteaux d'Amboise comme à Saumur. Cette décoration viticole (Legouy, 2012) rappelle la même ambiance paysagère des enluminures des « très riches heures du Duc de Berry » : ou comment le modèle historique devient mythe et est transformé en objet de marketing (figure 7). 


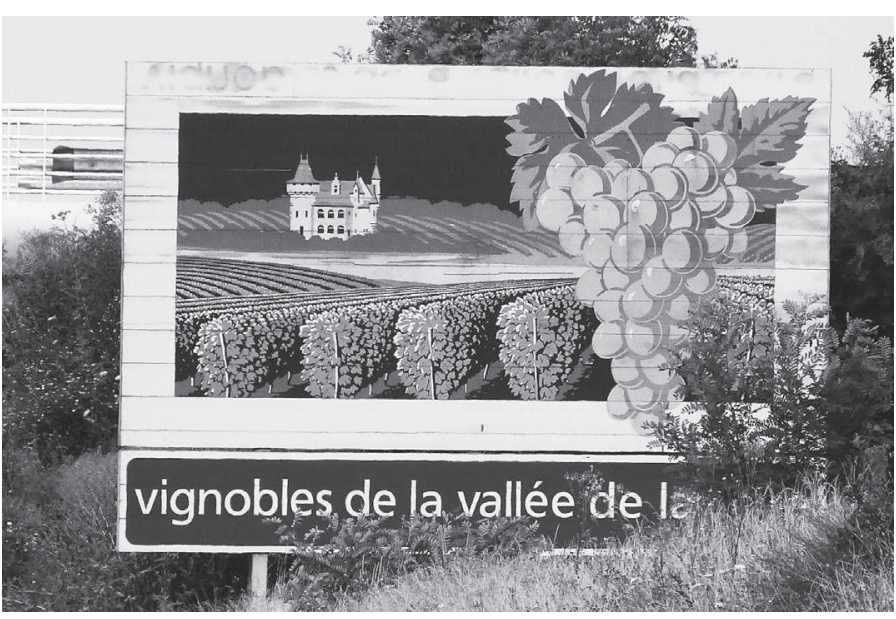

Figure 7 : Panneau touristique sur autoroute Tourist signpost on motorways

Cette image est-elle suffisante pour fonctionner en synergie et être efficace? La région a encore de la marge devant elle, comme le montre l'exemple de Valençay.

En région Centre - Val de Loire, les châteaux viticoles sont très peu nombreux. Pourtant, la relation avec le patrimoine gastronomique n'est pas établie ou très peu. Ils constituent paradoxalement un des éléments identitaires de la région. Prenons l'exemple de Valençay.

Le château y est l'emblème du Pays de Valençay. Cette petite région est sans doute la seule en France à proposer deux $\mathrm{AOC} / \mathrm{AOP}$ en vin et fromage avec le même nom. Nous sommes dans ce que Ph. Bourguignon (1997) a appelé l'accord parfait, c'està-dire une harmonie subtile et délicate entre un met et un vin. Les sommeliers, les œnologues comme les chefs cuisiniers préconisent volontiers un vin blanc (le Valençay) pour l'accompagnement des fromages de chèvre du Berry comme la pyramide de Valençay. Pourtant, la référence au château de ce territoire n'est pas spécialement explicite dans la promotion de ces deux produits gastronomiques. Certes, il se trouve bien quelques producteurs pour afficher sur les étiquettes de leurs bouteilles une reproduction visuelle du château, mais pas tous loin de là. Il est possible également de retrouver sur le site internet de ces deux AOP une discrète photographie du château, mais sans suite : la synergie entre ces trois éléments s'arrête rapidement. Le logo des AOC à l'entrée des communes situées dans l'aire de production de l'AOC viticole dressée à l'adresse des conducteurs et illisible à $50 \mathrm{~km} / \mathrm{h}$ exhibe un fromage, une grappe et un verre à vin, mais point de château.

S. Gatinel, alors directeur du Château de Valençay a expliqué en aparté que ce château était sous-utilisé pour la mise en valeur des deux AOC locales. Il était prêt à se servir de l'orangerie du château pour des dégustations et des animations gastronomiques si les acteurs et les responsables politiques lui donnaient le feu vert. L'argumentation est simple : « 100000 visiteurs par an viennent à Valençay, il serait possible de les intéresser au vin et au fromage par des animations et des expositions, leur proposer même d'acheter des produits de la région avant de repartir, comme dans les châteaux de la Loire où la fin de la visite dans la boutique des souvenirs est le passage obligé ${ }^{5}$.»

Le visiteur remarquera pourtant à la sortie de la gare de Valençay et de son aire d'accueil qui ont été complètement restaurées, quelques pieds de vignes plantés pour indiquer aux touristes l'importance de la vigne dans la région, mais pas de pancarte ou d'indication même sommaire sur l'accord vin - fromage - château. Le touriste ne retrouvera que très peu de mises en scènes paysagères évoquant les richesses patrimoniales de la région, peu de synergie œnotouristique avec le patrimoine monumental castral. La communication à Valençay est muette sur le sujet. On est dans le non-dit! Il en est autrement à Cheverny.

\section{UN EXEMPLE RÉCENT DE DÉVELOP- PEMENT TERRITORIAL ET DE LIAISON VIGNOBLE - CHÂTEAU : L'AOC Cheverny}

\section{L'AOC Cheverny et Cour-Cheverny, un passé princier}

Les vignobles de Cheverny et Cour-Cheverny constituent un excellent exemple des recompositions et des associations actuelles entre l'image du château et la vitiviniculture dans la vallée de la Loire. Ils présentent une parenté royale évidente autour de l'ancienne demeure de Diane de Poitiers, maitresse du roi Henri II. Mais ces vignobles ont connu les mêmes évolutions et le même destin que

5. Propos tenus en janvier 2010 sur le potentiel œnotouristique du château de Valençay. 
les autres vignobles ligériens, comme l'a très bien expliqué R. Dion (1934, 1952, 1959).

Les AOC Cheverny et Cour-Cheverny naissent en 1993 sur une vingtaine de communes au sud de Blois, en pleine Sologne viticole. Le cépage romorantin, utilisé pour les vins blancs de CourCheverny (les vins blancs chevernois utilisent le cépage sauvignon) a été apporté au début du $\mathrm{XVI}^{\mathrm{e}}$ siècle par François Ir à proximité de la maison royale éponyme. L'impulsion donnée à l'implantation de 80000 pieds de vigne dans la région provient du pouvoir central. La culture du raisin est confiée aux nobles et aux moines locaux, alors qu'une loi de 1577 du Parlement de Paris encourage les commerçants de vin à s'approvisionner plus au sud en direction des crus ligériens.

La vitiviniculture régionale a ensuite perdu de sa vivacité et de sa noblesse car les aristocrates furent très occupés à la cour du souverain. Nous pouvons identifier dans les vignobles de Cheverny et de Cour-Cheverny les mêmes problèmes rencontrés auprès des vignobles du Val de Loire d'Orléans à Tours. Les parcelles se morcellent et sont désormais travaillées par de «petits-cultivateurs-vignerons » (Legouy, 2012), dont les productions restent certes satisfaisantes quantitativement, mais pas qualitativement. Puis, à partir de la seconde moitié du $\mathrm{XIX}^{\mathrm{e}}$ siècle, les vignobles de Cheverny et de CourCheverny doivent, à l'image de l'ensemble des autres régions viticoles françaises, affronter la crise phylloxérique. Une politique volontariste permet alors d'arracher les plants perdus et de replanter rapidement, avec des hybrides, offrant de forts rendements mais une très mauvaise qualité vinicole. Il faut alors un long processus de re-concentration des parcelles les plus intéressantes et de suppression des moins fertiles, ainsi qu'une collaboration étroite avec les services de l'INAO pour reconstruire une vitiviniculture efficace et dynamique en pays chevernois. La définition d'une aire d'AOC en 1993 correspond ainsi à une renaissance, dont le nom Cheverny n'est pas étranger quant à l'association récurrente entre ces vignobles et l'image du château.

\section{Le vignoble et le château de Cheverny : une synergie réussie}

Une analyse récente a souligné la place centrale prise par le château dans l'image, la communication et la commercialisation des vins de Cheverny et de Cour-Cheverny (Vitré, 2011).

Tout d'abord, l'aire d'AOC s'est construite sur 532 ha tout autour du château. La principale puissance commerciale commune à tous ces vignerons est la maison des vins, dont les locaux se situent... à côté de l'entrée du modèle de Moulinsart, résidence du capitaine Haddock! L'objectif est donc clairement ici d'intégrer, dans une démarche œnotouristique et commerciale, les 300000 à 400000 visiteurs annuels du monument à la découverte puis à la consommation des vins locaux (figure 8).

Il semblait donc pertinent de rencontrer C. Couderc (entrevue du 13 mai 2011), gérante et animatrice de la Maison, qui a ouvert ses portes au printemps 2008. À l'image de l'AOC, la Maison des Vins est donc un outil jeune et dynamique, qui fonde son développement sur des outils neufs et innovants. Les 76000 touristes qui franchissent ses portes sont surtout curieux de découvrir le système des becs à dégustation, système novateur en France qui offre la possibilité à chaque connaisseur ou néophyte, selon la formule choisie (de 3 à 7 vins, « carte ambassadeur » par un initié accompagné d'amis), de goutter une centaine de références de vins blanc, rouge, rosé ou cour-cheverny (figure 9).

Un système de rotation dans la présentation des vins sur le module central ou dans les vins du jour permet une parfaite égalité entre les vignerons, qui peuvent s'auto-évaluer et se comparer lors d'une dégustation annuelle et anonyme des dix vins les

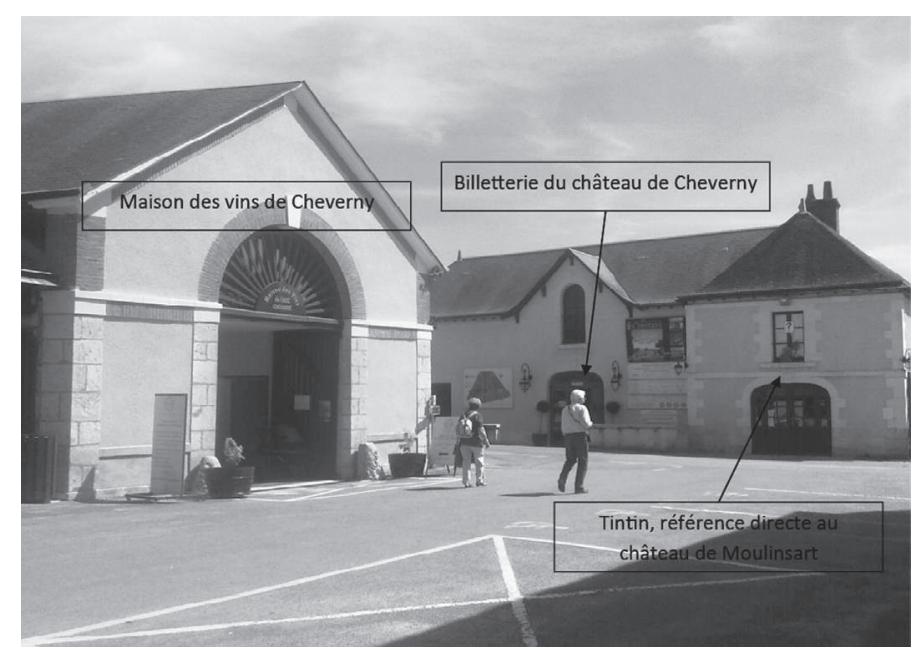

Figure 8 : La maison des vins de Cheverny Wine house in Cheverny 


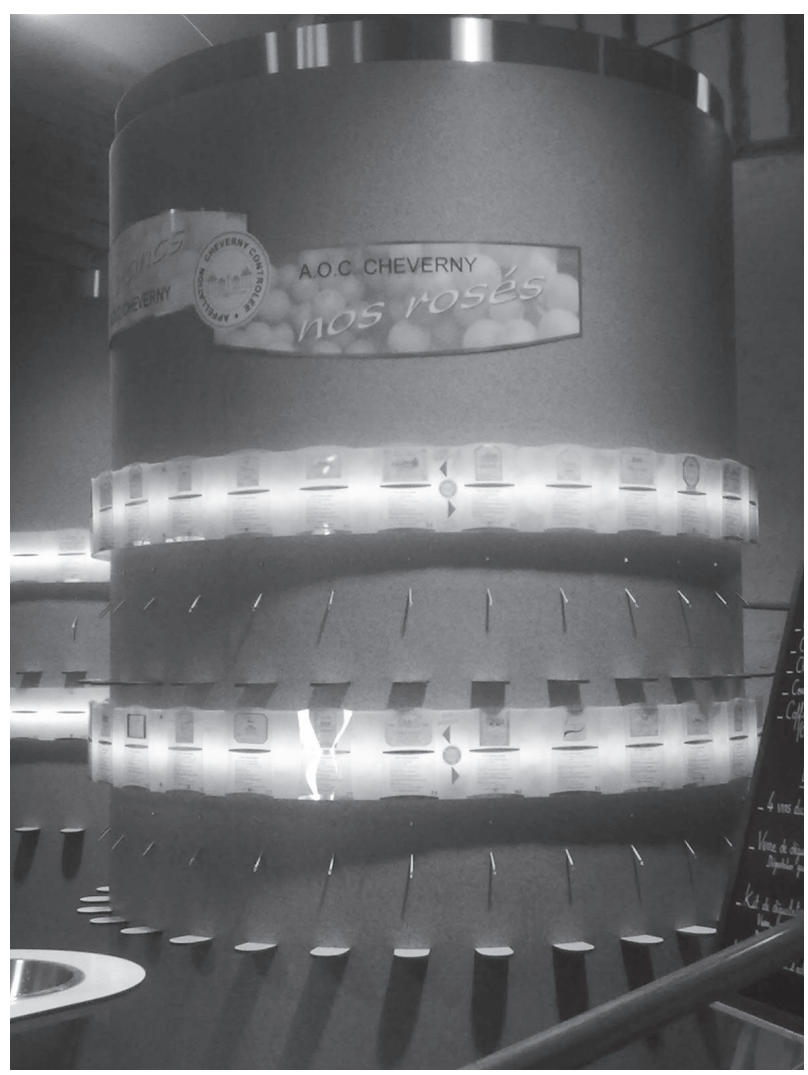

Figure 9 : Tour de dégustation avec ses becs verseurs Wine tasting tower

plus vendus. Les verres utilisés sont équipés d'une puce électronique permettant de collecter de précieuses statistiques : vins les plus essayés, rapport entre vins essayés et vins vendus, consommation limitée à $3 \mathrm{cl}$ par verre pour permettre à chacun de repartir en conduisant. Les ventes s'élèvent ainsi à 43243 bouteilles en 2010, soit la production d'une exploitation d'environ 10 à 15 ha. On rappellera ici en comparaison que 32 vignerons sont membres de Maison des vins et que la superficie moyenne par exploitation est de 8 ha sur l'ensemble du vignoble.

Les différents acteurs mobilisés se concentrent à présent sur le développement du site internet, où figurent la centaine de références présentée dans la Maison de vins (pour chaque vigneron, une référence de chaque couleur plus éventuellement un cour-cheverny). Les prix affichés sont les mêmes que ceux pratiqués individuellement par les vignerons. L'objectif est de capter des milliers de touristes, nationaux ou étrangers qui viennent, individuellement ou à travers des circuits touristiques, visiter l'un des plus beaux châteaux de la Loire.
À travers une démarche ludique et novatrice, ces potentiels futurs consommateurs vont découvrir un nouveau territoire viticole et prendre goût à des vins qui leur étaient probablement inconnus. L'objectif est donc alors de séduire et d'encourager, à travers une interface internet intuitive ou directement auprès des vignerons, une consommation chevernoise ou courchoise. Le château remplit ici le rôle d'épicentre d'où se diffusent les différents vecteurs de commercialisation et de territorialisation de notre AOC

L'identification au nom permet une ouverture aux touristes de passage, mais également aux potentiels consommateurs régionaux, nationaux et internationaux. Un amateur américain ou chinois peut facilement se construire sa propre géographie mentale en observant l'étiquette d'un vin de Cheverny où la silhouette du château est dans la plupart des cas représentée, puis en l'associant aux célèbres châteaux du Val de Loire, enviée, visitée et même à présent copiée (figure 10).

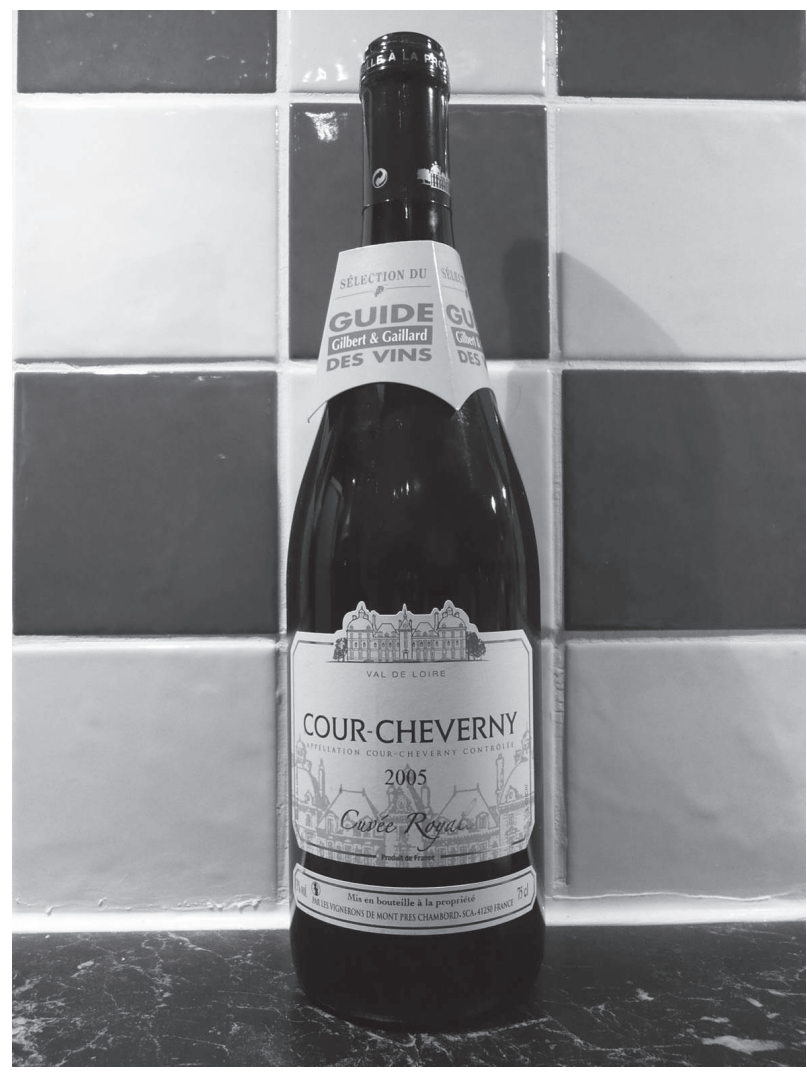

Figure 10 : Étiquette de Cheverny représentant le château The castle on Cheverny's label 
Les enjeux sont évidents. L'association vin-château semble être le meilleur vecteur de publicité pour plus de 70 opérateurs dispersés sur seulement 530 ha. Les quelques vignerons rencontrés expliquent les perspectives qu'offrent les pratiques œnotouristiques : visites de cave, escales des touropérateurs, projets de séjour à la ferme, sites internet et achat en ligne. Les diverses publications et mentions réalisées par les offices du tourisme locaux ou départemental visent encore une fois à vendre un produit identifiable par son histoire et son château.

Ces travaux ont également permis de mettre en relief une certaine volonté d'autonomisation des vignerons rencontrés avec le cadre pluriséculaire de l'ancienne résidence royale. Th. Puzelat, vigneron local sollicité par questionnaire, a par exemple apporté, à la question « quelle est selon vous l'image de marque de votre vin? », les réponses suivantes : «1-l'image du domaine, 2-l'image de l'AOC, 3-l'image de la région »...

L'association d'un vin et d'un château mondialement connu ne peut présenter que des avantages certains pour permettre et accompagner la naissance d'une AOC. Elle offre un nom, une histoire, une image de marque et des références comprises ou imaginées à travers le monde. Toutefois, il serait illusoire de limiter le travail de 72 opérateurs à une simple construction castrale. Le château de Chambord, proche de $40 \mathrm{~km}$ de son voisin chevernois, avait été dans les années 1990 proposé pour être associé aux vins de Cour-Cheverny et éviter ainsi que ce dernier ne soit, à tort, qu'un sous-produit du Cheverny. Mais cette anecdote démontre bien le lien ténu entre le château et le vin qui lui est associé, comme entre l'architecture paysanne typique du Val de Loire avec celle des châteaux.

Pourtant, si le château du Val de Loire et de la région Centre n'est plus et depuis longtemps accompagné de ses vignes, c'est aussi parce que la pression urbaine et l'étalement urbain ont joué leur rôle. Les châteaux, faut-il le rappeler, ne sont jamais éloignés des villes. Les aires d'AOC Cheverny et Cour-Cheverny sont également et particulièrement menacées par la périurbanisation qui se développe autour de l'agglomération de Blois. Il suffit en effet de se promener sur la rive gauche de la Loire pour compter le nombre de maisons et de lotissements en cours de construction en plein milieu du périmètre défini par l'INAO. Th. Puzelat déplore ainsi que la commune des Montils, à l'extrême-ouest de l'aire d'AOC, qui était à $75 \%$ recouverte par la vigne au XVIII ${ }^{\mathrm{e}}$ siècle, n'accueille plus que son frère et lui aujourd'hui comme derniers vignerons locaux.

Au-delà de la simple production vitivinicole et de sa commercialisation, les vignerons chevernois et courchois doivent donc se mobiliser et lutter contre les risques de disparition de leur premier outil de travail, la terre, face aux dangers du mitage. C'est dans cette optique que D. Tévenot, rencontré lors d'en entretien le 16 mars 2011, explique s'efforcer de produire, en plus de son raisin et de son vin, 25 ha de blé, d'orge et de tournesol, pour former une barrière face à ce processus urbain qui dénature les espaces vitivinicoles et supprime les traces d'activités anciennes mais présentes dans les mémoires collectives.

Les vignobles de Cheverny et de Cour-Cheverny atteignent donc le stade de la maturité. Le nom et l'histoire du château de Cheverny constituent leur lignage royal et marquent leur naissance. Ils sont et seront donc indissociables. Mais nous comprenons également que ses vignerons cherchent à s'autonomiser et à présenter aux consommateurs leurs propres produits, issus de leurs travaux et de leurs histoires personnels. Les vins de Cheverny et de Cour-Cheverny cherchent ainsi une seconde jeunesse en adoptant des pratiques plus durables et plus biologiques comme marqueurs identitaires. Leurs collaborations et leurs regroupements s'inscrivent de plus en plus au sein de réseaux comme ceux des Vignerons indépendants, du Groupement des Agriculteurs Biologiques du Loir-et-Cher (GABLEC) ou de l'Association des Vins Naturels $(\mathrm{AVN})$, et de plus en plus de bouteilles abandonnent l'empreinte du château sur leurs étiquettes. Nous pouvons par exemple identifier une vingtaine de producteurs en viticulture raisonnée sur les trente-deux membres de la Maison des Vins, et deux domaines sont actuellement en conversion en vignobles biologiques.

Le rappel du « château » pour les vins de Cheverny et de Cour-Cheverny est avant tout nominal et symbolique, et ne correspond pas aux paysages et aux organisations connues et reconnues dans le vignoble bordelais. Les associations à l'ancienne demeure de Diane de Poitiers ne se bornent qu'à un seul bâtiment, que nous retrouvons sur l'ensemble des étiquettes et qui constitue le cœur et le principal 
vecteur de diffusion de ce vignoble. Il constitue également la vitrine commerciale en installant à ses portes la maison des vins et le siège du syndicat des producteurs, récupérant ainsi des milliers de consommateurs locaux ou internationaux qui assurent la commercialisation et la diffusion de ces vins.

\section{Conclusion}

Pour conclure, il est possible d'affirmer que dans la région Centre - Val de Loire le processus de patrimonialisation et de territorialisation est une réalité, en témoigne une fréquentation touristique qui ne faiblit pas, mais que la synergie patrimoine castral - vignoble n'en est encore qu'à ses balbutiements. Dans cette région, il existe peu de châteaux viticoles en tant qu'exploitations viticoles qui restent l'apanage et l'invention du bordelais d'une part, et d'autre part, la liaison château - vignoble - vin est peu mise en œuvre. Le cas de Cheverny est une des rares exceptions qui confirme la règle. Les acteurs territoriaux, à commencer par ceux de la filière vitivinicole ne se sont pas encore approprié cette idée que le processus de patrimonialisation est vecteur de développement territorial. Si les Bordelais ont le potentiel en terme de patrimoine néo-castral au cœur de leurs champs de vignes, leurs pratiques œenotouristique peuvent encore prendre une ampleur plus grande, comme les professionnels du vin et du tourisme des autres régions viticoles ont encore beaucoup à apprendre en ce domaine. Le " château », élément patrimonial, qu'il soit architectural ou nominal, est un atout de développement économique et œenotouristique ainsi que le démontre la demande des Américains et qui a été repoussée récemment de mettre sur leurs étiquettes ce vocable.

Dans la région Centre les marques touristiques La Loire à vélo et Démarche d'excellence des grands sites $d u$ Val de Loire, ainsi que les quatre filières touristiques (patrimoine culturel, tourisme de nature, itinérances douces, art de vivre) ont tout à gagner à être reliées au monde de la vigne et du vin, comme le préconisent les expertises (2007) et la mission Val de Loire (2012) qui ont mis l'accent sur les bienfaits de la synergie œno-touristique.

\section{Bibliographie}

Bigando E., 2006. La synecdoque paysagère, une notion pour comprendre les représentations des paysages viticoles bourguignons et bordelais, Sud-Ouest Européen, n 21, p. 83-94.

BonTÉ Ch., 2009. Vivre les paysages inscrits au patrimoine mondial de l'Unesco, brochure de la Mission Val de Loire, patrimoine mondial, Tours, 23 p.

Bourguignon P., 1997. L'accord parfait, Paris, Éditions du Chêne, 184 p.

Chiva I., 1994. Une politique pour le patrimoine culturel rural, Rapport présenté au Ministre de la Culture et de la francophonie, Monsieur Jacques Toubon, 53 p.

[www.culture.gouv.fr/mpe/dossiers/documents/chiva.doc].

Collectif 2005. Rencontres internationales planète terroirs, Paris, Unesco, 2005, p. 66.

Combaud A., Legouy F., 2010. Roger Dion : un fervent partisan de l'analyse multiscalaire, Piтte Jean-Robert, Le bon vin. Entre terroir, savoir-faire et savoir-boire, Paris, CNRS éditions, p. 33-56.

Dion R., 1934. Le Val de Loire - étude de géographie régionale, Tours, Arrault \& Compagnie, 752 p.

Dion R., 1959 [rééd. 1977]. Histoire de la vigne et du vin en France, Flammarion, $570 \mathrm{p}$.

Dion R., 1990 [rééd.]. Le paysage et la vigne. Essais de géographie historique, Paris, Payot, 295 p.

Dubrule P., 2007. L'onotourisme, une valorisation des produits et $d u$ patrimoine vitivinicoles, Rapport remis au ministre de l'agriculture et au ministre du tourisme, Paris, $92 \mathrm{p}$.

Legouy F., 2007. Terroirs, AOC et qualité, Actes du colloque international Les terroirs, caractérisation, développement territorial et gouvernance, d'Aix-en-Provence (9-12 mai 2007)

Legouy F., 2008, La France vitivinicole dans la mondialisation, in Boulanger S., Legouy F., (dir.), 2008. Vins, vignes et vignerons en France et dans le monde, Historiens E Géographes, $\mathrm{n}^{\circ}$ 402, mai 2008, p. 123-151.

Legouy F., 2012. Les mises en scènes paysagères traditionnelles et nouvelles de la vigne et du vin, in WoLikow S. et JACQUet O., Territoires et terroirs $d u$ vin $d u$ XVIII $I^{e}$ au XXI ${ }^{e}$ siècles - Approche internationale d'une construction historique, Dijon, UB-EUD, p. 211-228.

Legouy F., 2012. Les héritages paysagers de la viticulture dans le Val de Loire d'Orléans à Tours et ses abords périphériques, Projets de paysage, 04-01-2012. [http://www. projetsdepaysage.fr/fr/les_heritages_paysagers_de_la_viticulture_dans_le_val_de_loire_d_orleans_a_tours_et_ses_abords_ peripheriques].

Lignon-Darmaillac S., 2009. L'œenotourisme en France, nouvelle valorisation des vignobles - analyse et bilan, Bordeaux, Féret, 255 p.

Péron F., 2001. Patrimoine culturel et géographie sociale, Faire la géographie sociale aujourd'hui, Caen, Presses universitaire de Caen, p. 19-30.

Pinchemel P., (dir.), 1980. La France. Activités, milieux ruraux et urbains, Paris, Armand Colin.

Pitte J.-R., 1990. Le mariage d'amour de la France et du vin, L'Histoire, $\mathrm{n}^{\circ} 136$, p. 88-90. 
RAstoin J.-L., 2004. Quelle stratégie pour les produits de terroir dans un contexte de globalisation des marché?, Cafés débats à Marciac, p. 8.

RÉJALOT M., 2003. Le modèle viti-vinicole bordelais dans sa filière (1980-2003). Un idéal français dans la tourmente?, Thèse de géographie de l'Université de Bordeaux 3 sous la direction de Philippe Roudié, 372 p. + annexes.

RÉjalot M., 2011 . Des châteaux en Gironde, Le Festin, nº hors série : au cœur des vins de Bordeaux et du Sud-Ouest, p. 60-65.

Roudié Ph., 2000. Vignobles et vins de France, élitisme et diversité, Historiens E Géographes, n 370, p. 341-346.

Roudié Ph., 2000. Vous avez dit « château »? Essai sémantique d'un modèle viticole venu du bordelais, Annales de Géographie, n 614-615, Paris, Armand Colin, p. 415-425.
SENIL N., 2011. Une reconstruction de l'espace-temps : approche croisée des processus de patrimonialisation et de territorialisation dans les territoires ruraux en France et au Maroc, Thèse de doctorat de géographie, Université de Grenoble, 562 p

Sylvander Bertil (dir.), 2005. Les signes officiels de qualité et d'origine européens. Quelle insertion dans une économie mondialisée?, Économie rurale, ${ }^{\circ} 299$.

Vitré Ch., 2011. Les vignobles de Cheverny et de Cour-Cheverny, Mémoire de recherche de Master, Université d'Orléans, $50 \mathrm{p}$. 